\title{
Strain engineering of electronic and optical properties of monolayer diboron dinitride
}

\author{
Salih Demirci $\odot,{ }^{1,2}$ Soheil Ershad $\operatorname{Rad} \odot,{ }^{1}$ and Seymur Jahangirov $\odot^{1, *}$ \\ ${ }^{1}$ UNAM-Institute of Materials Science and Nanotechnology, Bilkent University, Ankara 06800, Turkey \\ ${ }^{2}$ Department of Physics, Kırıkale University, Kırıkkale 71450, Turkey
}

(Received 23 December 2020; revised 16 November 2021; accepted 17 November 2021; published 29 November 2021)

\begin{abstract}
We studied the effect of strain engineering on the electronic, structural, mechanical, and optical properties of orthorhombic diboron dinitride $\left(\mathrm{o}-\mathrm{B}_{2} \mathrm{~N}_{2}\right)$ through first-principles calculations. The 1.7-eV direct band gap observed in the unstrained $\mathrm{o}-\mathrm{B}_{2} \mathrm{~N}_{2}$ can be tuned up to $3 \mathrm{eV}$ or down to $1 \mathrm{eV}$ by applying $12 \%$ tensile strain in armchair and zigzag directions, respectively. Ultimate strain values of $\mathrm{o}-\mathrm{B}_{2} \mathrm{~N}_{2}$ were found to be comparable with that of graphene. Our calculations revealed that the partial alignment of the band edges with the redox potentials of water in pristine o- $\mathrm{B}_{2} \mathrm{~N}_{2}$ can be tuned into a full alignment under the armchair and biaxial tensile strains. The anisotropic charge carrier mobility found in $\mathrm{o}-\mathrm{B}_{2} \mathrm{~N}_{2}$ prolongs the average lifetime of the carrier drift, creating a suitable condition for photoinduced catalytic reactions on its surface. Finally, we found that even in extreme straining regimes, the highly anisotropic optical absorption of o- $\mathrm{B}_{2} \mathrm{~N}_{2}$ with strong absorption in the visible range is preserved. Having strong visible light absorption and prolonged carrier migration time, we propose that strain engineering is an effective route to tune the band gap energy and band alignment of o- $\mathrm{B}_{2} \mathrm{~N}_{2}$ and turn this two-dimensional material into a promising photocatalyst for efficient hydrogen production from water splitting.
\end{abstract}

DOI: 10.1103/PhysRevB.104.205432

\section{INTRODUCTION}

Since the revolutionary advent of graphene [1-4] in 2004, two-dimensional (2D) materials have attracted intensive interest from researchers. In 2D structures, the out-of-plane quantum confinement gives rise to a plethora of exotic properties, unprecedented in their bulk counterparts. In graphene, the presence of Dirac cones at corners of the Brillouin zone induces semimetallic charge transport behavior [5], making graphene inapplicable in semiconductor devices. Moreover, very poor light absorption in a single sheet of graphene deteriorates its functionality in optoelectronic applications [6]. These deficiencies have motivated scientists to systematically search for new 2D materials and employ a variety of approaches to tailor their undesired properties. In the case of graphene, for instance, techniques such as doping [7], charging [8], making heterostructures [9], or even changing the chemistry by hydrogenation [10] are widely employed to create superior features.

Strain engineering is another conventional method to manipulate the structural, electronic, and optical properties of 2D materials. Several experimental approaches are available to apply tensile and compressive strains to $2 \mathrm{D}$ structures. The monolayer can be transferred on top of a flexible substrate, and uniaxial strain can be induced by bending the substrate [11]. In addition to bending, the substrate can also be stretched to induce a homogeneous uniaxial tensile strain [12]. Another approach is to exploit the thermal expansion mismatch between the substrate and the monolayer to induce a uniform biaxial strain [13]. Finally, a clever approach is to exploit

\footnotetext{
*seymur@unam.bilkent.edu.tr
}

substrates with the piezoelectric feature, which enables fine control over the scope of strain through applying a precise electric potential to the piezoelectric material, making both tensile and compressive strains achievable $[14,15]$. The strain tunability of many 2D systems, such as graphene [11,16] and transition metal dichalcogenides (TMDs) [12-14,17], was studied with these approaches. The maximum strain that can be applied using these methods is less than 5\% [18]. However, by employing modern approaches, such as fabrication of microelectromechanical system (MEMS) devices, strains of more than $10 \%$ can be applied to 2D structures [19]. Motivated by the development of experimental techniques employed in strain engineering, first-principles approaches were widely utilized to predict the strain-induced response of many 2D systems, including graphene [20], graphane [21], transition metal dichalcogenides [22], etc.

The abundance and thermal stability of 2D boron nitride compounds have turned them into suitable candidates for many applications. Hexagonal boron nitride (h-BN) is the most conventional and stable $2 \mathrm{D}$ form of the boron nitride compounds [23]. A large band gap makes h-BN an insulator [24]. In addition, h-BN light absorption mainly takes place in the range of 5-6 eV [25], which is far out of the visible region, making it highly transparent. These drawbacks provide room for the investigation of other 2D polymorphs of boron nitride. Recently, another stable 2D boron nitride compound, known as o- $\mathrm{B}_{2} \mathrm{~N}_{2}$ [26], was predicted through first-principles calculations, where B-B and $\mathrm{N}-\mathrm{N}$ pairs connect to create an orthorhombic unit cell with a space group symmetry of Pmmm (No. 47), a direct band gap of $1.7 \mathrm{eV}$, and highly anisotropic optical absorption in the visible range of the light spectrum, suitable for optoelectronic applications. 
In this paper, inspired by the strong visible light absorption in o- $\mathrm{B}_{2} \mathrm{~N}_{2}$ and its suitability for optoelectronic applications, we studied the effect of uniaxial and biaxial strain engineering on the electronic and optical properties of o- $\mathrm{B}_{2} \mathrm{~N}_{2}$. To make sure that this structure can tolerate extreme strains without losing its integrity, the ultimate tensile strength and its corresponding ultimate strain were calculated. The strain-induced response of the structural properties, including bond lengths and lattice parameters, was probed. We found that the $1.7-\mathrm{eV}$ band gap in the pristine condition can be modified broadly to create a suitable condition for photocatalytic applications [27-33]. Finally, the optical absorption response under strain was studied. We found that the anisotropic optical absorption of $o-B_{2} N_{2}$ in the visible range remains intact under a wide range of strain conditions.

\section{METHODS}

We performed first-principles calculations based on density functional theory (DFT) using a plane-wave basis set with an energy cutoff of $520 \mathrm{eV}$. We utilized projector-augmented wave potentials [34], and the exchange-correlation potential is approximated by generalized gradient approximation (GGA) with the Perdew, Burke, and Ernzerhof (PBE) functional [35]. The Brillouin zone (BZ) is represented by an $8 \times 16 \times 1 \mathrm{k}$-point grid in the Monkhorst-Pack scheme [36]. The equilibrium configuration of atoms is determined by minimizing the total energy of the system using the conjugate gradient method. The energy convergence criteria between the two consecutive electronic and ionic steps are taken to be $10^{-5}$ and $10^{-4} \mathrm{eV}$, respectively. In addition, the maximum pressure on the lattice has been lowered down to $0.1 \mathrm{kbar}$. To avoid interaction between periodic images in adjacent cells, a vacuum spacing of at least $20 \AA$ was used. All calculations were performed using the Vienna $a b$ initio simulation package (VASP) [37]. Electronic structure calculations were corrected via HeydScuseria-Ernzerhof (HSE06) hybrid functionals [38]. The stress-strain curves are calculated, using a $3 \times 3 \times 1$ supercell. To calculate the optical response of the structure to strain, the imaginary part of the frequency-dependent dielectric function, which corresponds to optical absorption, is calculated using the random phase approximation (RPA) with $112 \times 224 \times 1 \mathrm{k}$ points and 56 bands. The local field effects are included at the Hartree level only [39]. Results obtained at the PBE level are then corrected using the scissors operation based on the difference between the PBE and HSE band gaps.

\section{RESULTS AND DISCUSSION}

o- $\mathrm{B}_{2} \mathrm{~N}_{2}$ has an orthorhombic unit cell, with two inequivalent lattice constants of $a=4.57 \AA$ and $b=2.50 \AA$. The atomic configuration of $o-B_{2} N_{2}$ is shown in Fig. 1(a). The structure is made of $\mathrm{B}-\mathrm{B}$ and $\mathrm{N}-\mathrm{N}$ dimers which are connected to each other to create an orthorhombic honeycomb structure. In an unstrained structure, B-N, B-B, and N-N bond lengths are $1.44,1.44$, and $1.70 \AA$, respectively, where the B-N length is equivalent to the conventional $\mathrm{h}$ - $\mathrm{BN}$ counterpart. In the pristine condition, $\mathrm{o}-\mathrm{B}_{2} \mathrm{~N}_{2}$ is a semiconductor with a direct band gap of $1.70 \mathrm{eV}$, according to HSE06 calculations, as can be seen in Fig. 1(b) [26].
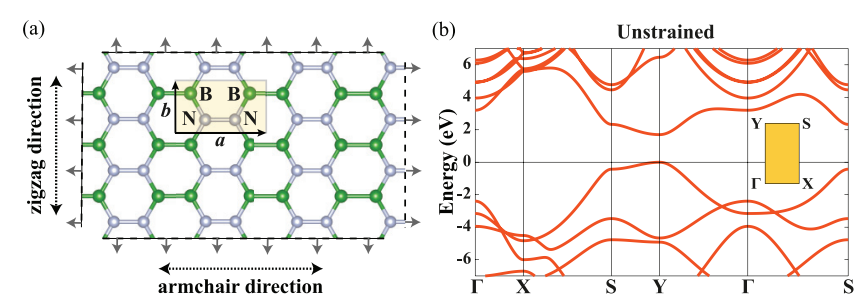

(c)
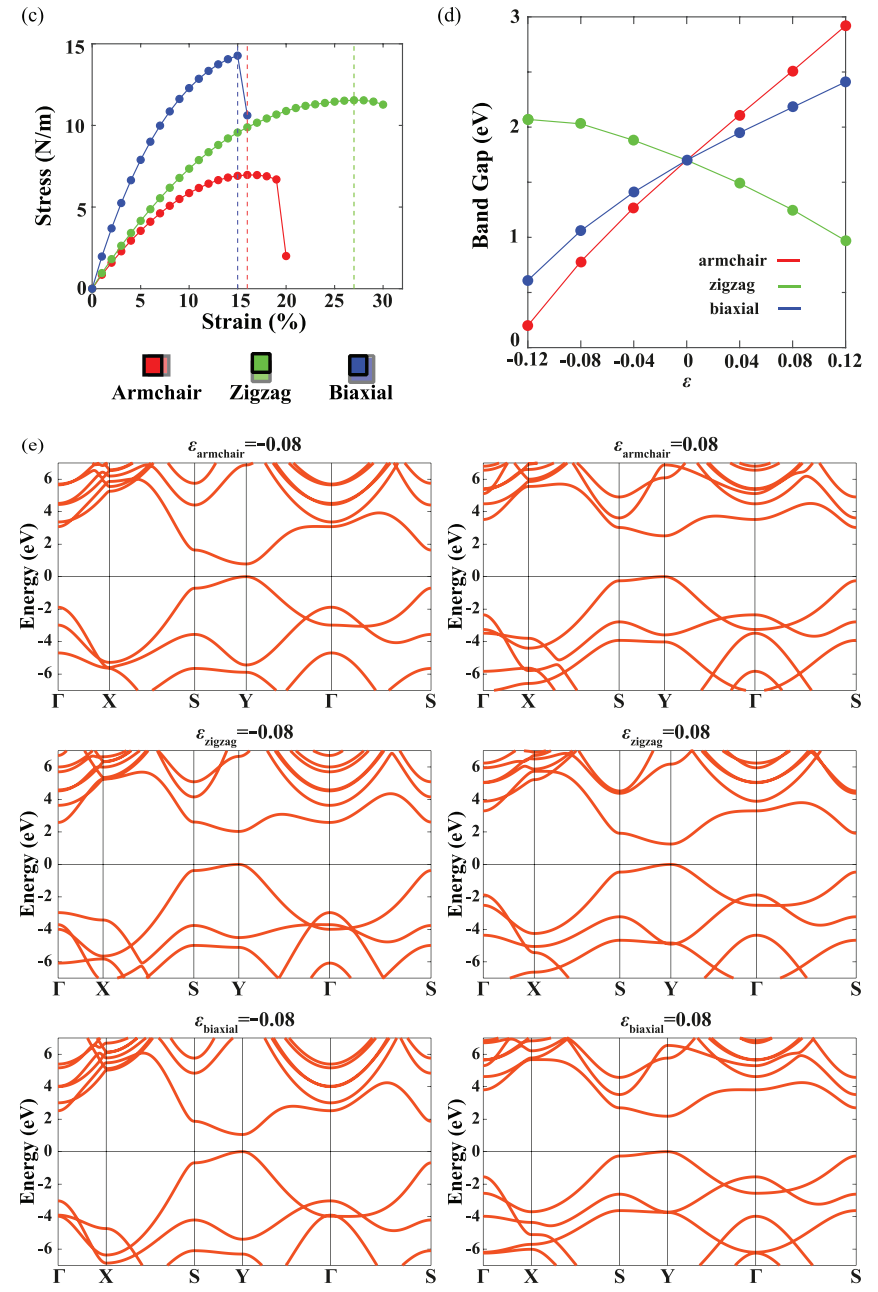

FIG. 1. (a) Ball-and-stick model of o- $\mathrm{B}_{2} \mathrm{~N}_{2}$, where gray and green balls denote nitrogen and boron atoms, respectively. The unit cell is depicted in a yellow shade. (b) Electronic band structure of $\mathrm{o}-\mathrm{B}_{2} \mathrm{~N}_{2}$ in the pristine condition (calculated using the HSE06 hybrid functional). The orange inset shows the high-symmetry lines of the BZ. (c) Stress-strain curves of o- $\mathrm{B}_{2} \mathrm{~N}_{2}$ for armchair (red), zigzag (green), and biaxial (blue) strains, where the vertical dashed lines show the percentage of ultimate strain. (d) Tunability of the direct band gap of o- $\mathrm{B}_{2} \mathrm{~N}_{2}$ with respect to the applied strain. (e) Same as (b) for various strain conditions.

In 2D materials, an applied strain can be described by the following expression:

$$
\varepsilon=\left(l-l_{0}\right) / l_{0},
$$

where $l$ and $l_{0}$ are strained and equilibrium unit-cell lengths along the strain direction, respectively. Based on Eq. (1), uniaxial- $x$ (armchair), uniaxial- $y$ (zigzag), and biaxial strains were applied to $\mathrm{o}-\mathrm{B}_{2} \mathrm{~N}_{2}$. The stress-strain curve of $\mathrm{o}-\mathrm{B}_{2} \mathrm{~N}_{2}$ 
TABLE I. The evolution of band gap, bond lengths, electron affinity EA under armchair, zigzag, and biaxial strained conditions.

\begin{tabular}{|c|c|c|c|c|c|c|c|}
\hline$\varepsilon$ & $\begin{array}{c}\text { Unstrained } \\
0\end{array}$ & -0.12 & +0.12 & -0.12 & +0.12 & -0.12 & +0.12 \\
\hline$a(\AA)$ & 4.57 & 4.02 & 5.12 & 4.68 & 4.50 & 4.02 & 5.12 \\
\hline$b(\AA)$ & 2.50 & 2.57 & 2.48 & 2.20 & 2.80 & 2.20 & 2.80 \\
\hline B-B (̊̊) & 1.70 & 1.50 & 2.02 & 1.69 & 1.70 & 1.42 & 2.03 \\
\hline Second B-B (尺) & 2.87 & 2.52 & 3.10 & 2.99 & 2.80 & 2.60 & 3.09 \\
\hline Second N-N $(\AA)$ & 3.13 & 2.68 & 3.56 & 3.29 & 3.04 & 2.76 & 3.47 \\
\hline $\mathrm{EA}(\mathrm{eV})$ & 3.05 & 3.77 & 2.54 & 1.92 & 3.90 & 2.59 & 3.42 \\
\hline
\end{tabular}

under various types of strain is depicted in Fig. 1(c). We found the ultimate tensile strength (UTS) of $\mathrm{o}-\mathrm{B}_{2} \mathrm{~N}_{2}$ under biaxial strain to be $14.3 \mathrm{~N} / \mathrm{m}$, which is nearly half of the UTS value that we found for graphene $(32.0 \mathrm{~N} / \mathrm{m})$ and $\mathrm{h}-\mathrm{BN}$ $(27.8 \mathrm{~N} / \mathrm{m})$ in agreement with the literature [40,41]. This places $\mathrm{o}-\mathrm{B}_{2} \mathrm{~N}_{2}$ among the $2 \mathrm{D}$ materials with relatively high UTS. The strength of a $2 \mathrm{D}$ material has a close relation with the charge transfer among its bonding atoms. Our Bader charge analysis shows that the charge transfer between boron and nitrogen atoms in $\mathrm{o}-\mathrm{B}_{2} \mathrm{~N}_{2}$ is half that in h-BN, which justifies the inferior UTS and ultimate strain of o- $\mathrm{B}_{2} \mathrm{~N}_{2}$. However, with ultimate strains of 15,16 , and $28 \%$ for biaxial, armchair, and zigzag strain regimes, $\mathrm{o}-\mathrm{B}_{2} \mathrm{~N}_{2}$ is still among the $2 \mathrm{D}$ materials that can tolerate extreme strains. The lower ultimate strain under the armchair and biaxial strains is mainly due to the weakness of B-B bonds compared with the B-N bonds, where after the UTS point B-B bonds get detached. Nonetheless, these ultimate strain values are comparable with that of graphene (22\% for biaxial) and h-BN (24\% for biaxial) $[40,41]$. During the strain-engineering process of a $2 \mathrm{D}$ material, extreme strains can be imposed on the structure. A suitable candidate for strain engineering should be able to maintain its integrity under severe deformation. Although due to the technical constraints the maximum strain that has been applied to 2D materials is around $10 \%$, the high ultimate strains found in $\mathrm{o}-\mathrm{B}_{2} \mathrm{~N}_{2}$ satisfy the prerequisites of strain engineering beyond this threshold in the future.

Assuring that $\mathrm{o}-\mathrm{B}_{2} \mathrm{~N}_{2}$ is a suitable candidate for strain engineering of its properties, we further analyzed the effect of the strain on its electronic properties. For the sake of comparison, the band structure of $\mathrm{o}-\mathrm{B}_{2} \mathrm{~N}_{2}$ in the pristine condition is presented in Fig. 1(b), and the strain-engineered band structures for the two exemplary cases of $\varepsilon=-0.08$ and $\varepsilon=+0.08$ are presented in Fig. 1(e). The direct band gap of $\mathrm{o}-\mathrm{B}_{2} \mathrm{~N}_{2}$ is created by the dispersion in both valence and conduction band edges along the $S-Y$ direction. Our tight-binding analyses reveal that the dispersion in the conduction and valence is created by the interaction between the second-neighbor B-B and N-N atoms along the armchair direction, respectively [26]. Applying tensile strain along the armchair direction diminishes these interactions and results in less dispersive bands and a larger band gap, while compressive strain has the opposite effect. The reverse trend is observed in the case of the uniaxial compressive and tensile strain along the zigzag direction. This is because $\mathrm{o}-\mathrm{B}_{2} \mathrm{~N}_{2}$ has a positive
Poisson's ratio: Applying uniaxial tensile strain in the zigzag direction lowers the distance while increasing the interaction between the second-neighbor B-B and N-N atoms along the armchair direction. Biaxial strain creates behavior similar to the armchair case although the variation is less pronounced. This can be corroborated by the fact that under biaxial tensile (compressive) strain the distances of second-neighbor B-B and $\mathrm{N}-\mathrm{N}$ atoms increase (decrease), but not as much as they do in uniaxial tensile (compressive) strain along the armchair direction (see Table I).

The variation in the band gap for all cases of compressive and tensile strain is shown in Fig. 1(d). Note that in all strain regimes the band gap can be tuned over a broad energy span. The most prominent tunability occurs under armchair strain, where the band gap variation covers a $3-\mathrm{eV}$ zone. This scope of strain tunability is one of the exotic features of $\mathrm{o}-\mathrm{B}_{2} \mathrm{~N}_{2}$, which is rare among conventional 2D materials. For instance, in graphene, which is a semimetal in its unstrained condition, the band gap can be opened up to $0.5 \mathrm{eV}$ by applying up to $15 \%$ uniform tensile strain along the armchair direction, while zigzag tunability is much lower [20]. The band gap of silicene and germanene cannot be tuned significantly through uniform strain, where the band gap increases (decreases) on the order of meV upon applying tensile (compressive) strain [42-44]. Unstrained antimonene has a band gap around $0.8 \mathrm{eV}$, while up to $4 \%$ armchair strain can widen this band gap to $1.1 \mathrm{eV}$; further straining decreases the band gap energy and limits the scope of tunability [45]. TMDs have a direct band gap with the gap located at the $K$ point of the BZ, where the band gap varies depending on types of elements around 1-2 eV. Uniaxial or biaxial tensile strains linearly decrease the gap, while inducing a direct to indirect transition in small strains $[22,46]$. At high tensile strains of more than $12 \%$, the band gap eventually closes, without breaking the bonds [47]. TMDs exhibit the same behavior upon the compressive strain, but the tunability is much lower than the tensile strain [48]. Firstprinciples studies of phosphorene show that the electronic and optical gap increases by $0.2 \mathrm{eV}$ upon applying uniaxial tensile strain up to $5 \%$ and decreases by $0.5 \mathrm{eV}$ in the case of uniaxial compressive strain up to $10 \%$ and that a direct to indirect transition takes place around 5\% uniaxial tensile strain [49-51]. A monolayer of h-BN has an insulator nature with a wide band gap of $4.7 \mathrm{eV}$; through biaxial tensile strain, the gap can be reduced to less than $2 \mathrm{eV}$ [52], but this is only achievable in extreme strains that are higher than $25 \%$. In 
comparison to the aforementioned $2 \mathrm{D}$ materials, $\mathrm{o}-\mathrm{B}_{2} \mathrm{~N}_{2}$ has a promising band gap tunability. To accentuate this feature of $\mathrm{o}-\mathrm{B}_{2} \mathrm{~N}_{2}$, we extracted the slope of the band-gap-vs-strain curve for various conventional 2D materials, assuming a linear band gap variation around the equilibrium. While in $\mathrm{o}-\mathrm{B}_{2} \mathrm{~N}_{2}$ this slope is around $100 \mathrm{meV}$ per armchair strain percentage, in graphene the maximum of this slope is around $25 \mathrm{meV}$ per armchair strain percentage, in silicene this slope is less than $5 \mathrm{meV}$ per armchair strain percentage, and in antimonene and h-BN this value is around $75 \mathrm{meV}$ per biaxial strain percentage. In TMDs, such as $\mathrm{MoSe}_{2}$ the slope is close to that of o- $\mathrm{B}_{2} \mathrm{~N}_{2}$, and in $\mathrm{WS}_{2}$ and $\mathrm{MoS}_{2}$ this slope is even higher than that of o- $\mathrm{B}_{2} \mathrm{~N}_{2}$; however, in these compounds, the direct to indirect transition occurring around $2 \%$ biaxial tensile strain deteriorates the effectiveness of strain manipulation for optoelectronic applications. Moreover, deformation potentials found in $\mathrm{o}-\mathrm{B}_{2} \mathrm{~N}_{2}$, which based on Eq. (4) have a direct relation to the slope of the band gap variation near the equilibrium point, show higher values compared with graphene, h-BN, phosphorene, arsenene, antimonene, and silicene [53-55].

Another important feature that appears in $\mathrm{o}-\mathrm{B}_{2} \mathrm{~N}_{2}$ upon strain engineering, is its anisotropic band tunability. In comparison to compressive strain, it is easier to apply tensile strain to a $2 \mathrm{D}$ material, and one can go higher in the percentage of applied strain. Thus direction-dependent tunability of $\mathrm{o}-\mathrm{B}_{2} \mathrm{~N}_{2}$ enables both widening and narrowing of band gap energy over a wide range (0.97-2.92 eV) only through applying tensile strain. Although direction-dependent band gap tunability has already been reported in several 2D materials with intrinsic structural anisotropy such as tetrahex structures (for instance, tetrahex-carbon, tetrahex-SiC, and tetrahex-BN all exhibit this feature with less than 1.0-, 0.5-, and 0.8-eV tunability over $10 \%$ tensile strain, respectively [56-58]), the $\sim 2-\mathrm{eV}$ anisotropic tunability found in $\mathrm{o}-\mathrm{B}_{2} \mathrm{~N}_{2}$ makes it outstanding.

The response of the bond lengths and lattice parameters to strain is demonstrated in Table I. Since o- $\mathrm{B}_{2} \mathrm{~N}_{2}$ has a positive Poisson's ratio, in the case of uniaxial strain, the lattice constant transverse to the axis of strain always changes inversely compared with the axial lattice constant. In o- $\mathrm{B}_{2} \mathrm{~N}_{2}, \mathrm{~B}-\mathrm{B}$ and $\mathrm{N}-\mathrm{N}$ bonds are aligned in the armchair direction. Therefore the B-B and N-N bond lengths increase to compensate for the strain in this direction, while the B-N bond length is almost unaffected during the armchair strain. In addition, the increase in the second-neighbor B-B and N-N distances along the armchair direction shows that a part of the elongation is compensated by the rotation of $\mathrm{B}-\mathrm{N}$ bonds in a way that the B-N-B and N-B-N angles increase along the armchair direction. The B-N bonding energy is around $500 \mathrm{~kJ} / \mathrm{mol}$, which is significantly higher than that of $\mathrm{B}-\mathrm{B}(310 \mathrm{~kJ} / \mathrm{mol})$ and N-N (160 kJ/mol); consequently, the stiffer B-N bonds are less prone to elongation. On the other hand, none of the bonds is oriented along the zigzag direction; there are only zigzag chains of B-N bonds aligned in this direction that need to compensate for the strain. Hence the length of B-B and N-N bonds remains almost unchanged during uniaxial strain along the zigzag direction, while B-N bond lengths increase. The second-neighbor B-B and N-N distances (along the armchair direction) show a decreasing trend that indicates the widening of angles to neutralize the zigzag strain. Finally, in the case of biaxial strain, the lattice expands in a way that all bonds get stretched, and the second-neighbor distances also increase.

Excitonic average lifetime plays a crucial role in photocatalytic efficiency. To achieve better insight into the electron-hole separation in $\mathrm{o}-\mathrm{B}_{2} \mathrm{~N}_{2}$, we investigated the charge carriers' effective masses and mobilities. To study the effective mass, we fit the following equation to the conduction band minimum (CBM) for electrons and valence band maximum (VBM) for holes, calculated by the HSE06 hybrid functional:

$$
m^{*}=\hbar^{2}\left(\frac{d^{2} E(k)}{d k^{2}}\right)^{-1}
$$

The calculated effective masses of electrons and holes, along the $Y S\left(m_{x}\right)$ and $Y \Gamma\left(m_{y}\right)$ edges of the BZ, are reported in Table II. One can see that the effective mass values are highly anisotropic in $\mathrm{o}-\mathrm{B}_{2} \mathrm{~N}_{2}$. Having the effective masses, we further calculated the charge carrier mobilities along the $Y S$ (denoted by $\mu_{x}^{2 \mathrm{D}}$ ) and $Y \Gamma$ (denoted by $\mu_{y}^{2 \mathrm{D}}$ ) directions via the following expression [59]:

$$
\mu_{i}=\frac{e h^{3}\left(\frac{5 C_{i}+3 C_{i}}{8}\right)}{K_{B} T\left(m_{i}\right)^{\frac{3}{2}}\left(m_{j}\right)^{\frac{1}{2}}\left(\frac{9 E_{1 i}^{2}+7 E_{1 i} E_{1 j}+4 E_{1 j}^{2}}{20}\right)},
$$

where $i$ determines the transport direction and $m_{i}$ is the effective mass along direction $i$. The temperature is assumed to be $T=300 \mathrm{~K}$. The $E_{1 i}$ is the deformation potential constant of the charge carrier, obtained from the following expression:

$$
E_{1 i}=\frac{\Delta E}{\left(\frac{\Delta l}{l_{0}}\right)}
$$

where $\Delta E$ is the variation in the energy of the CBM and VBM bands under small strains ( $\varepsilon=0.5 \%$ was considered). The calculated values for $E_{1 i}$ are listed in Table II. The $C_{i}$ is the in-plane stiffness constant, calculated using the following formula and presented in Table II:

$$
C_{i}=2\left(\frac{E_{i}-E_{0}}{A_{0}}\right)\left(\frac{\Delta l}{l_{0}}\right)^{-2},
$$

where $E_{i}$ is the total energy of one unit cell after deformation, $E_{0}$ is the equilibrium total energy, and $A_{0}$ is the area of one unit cell. Previous studies showed that Eq. (3) tends to underestimate the absolute value of charge carrier mobility. Regarding the mobility ratio, however, Eq. (3) yields values close to experimental results [60]. Recently, Zhao et al. [61] calculated the mobility values for o- $\mathrm{B}_{2} \mathrm{~N}_{2}$, using the modified BardeenShockley formula. That formula tends to overestimate both the mobility values and the mobility ratios, which accounts for the differences in our results. We expect the experimental values for mobility to be higher than our results, and the mobility ratio to be close to our calculations. Our analysis shows that the charge carrier mobilities found in $\mathrm{o}-\mathrm{B}_{2} \mathrm{~N}_{2}$ are highly anisotropic. This feature prolongs the excitonic separation and creates a suitable condition for photocatalytic applications.

We further investigated the effect of strain engineering on the electron affinity (EA) of o- $\mathrm{B}_{2} \mathrm{~N}_{2}$. In practice, EA is of crucial importance in electron-emitting and photosensitive applications. In the semiconductor materials, the electron affinity is defined as the energy required to take an electron 
TABLE II. Charge carrier properties based on HSE06 calculations. Here, $m_{i}$ is the effective mass, $C_{i}$ is the elastic constant in N/m, $E_{1}^{i}$ is the deformation potential constant in $\mathrm{eV}$, and $\mu_{i}^{2 \mathrm{D}}$ is the carrier mobility at $T=300 \mathrm{~K} \mathrm{in} \mathrm{cm}^{2} \mathrm{~V}^{-1} \mathrm{~s}^{-1}$, while $i=x$ and $i=y$ correspond to armchair and zigzag directions, respectively.

\begin{tabular}{lcccccccc}
\hline \hline Carrier type & $m_{x}$ & $m_{y}$ & $C_{x}$ & $C_{y}$ & $E_{1}^{x}$ & $E_{1}^{y}$ & $\mu_{x}^{2 \mathrm{D}}$ & $\mu_{y}^{2 \mathrm{D}}$ \\
\hline Electron & 1.53 & 0.49 & 240 & 273 & 4.22 & 8.56 & 114 & 265 \\
Hole & 1.77 & 0.47 & 240 & 273 & 6.34 & 3.80 & 113 & 567 \\
\hline \hline
\end{tabular}

from the conduction band edge into the vacuum [62],

$$
\mathrm{EA}=E_{\mathrm{vac}}-E_{\mathrm{cbe}},
$$

where the vacuum energy $E_{\mathrm{vac}}$ is extracted from the asymptotic value of the electrostatic potential as one moves far away in the $z$ direction. The response of EA to strain is reported in Table I. One can see that strain engineering offers tunability of the electron affinity in a wide range from $1.92 \mathrm{eV}$ up to $3.90 \mathrm{eV}$.

In the pristine condition, o- $\mathrm{B}_{2} \mathrm{~N}_{2}$ offers a polarized optical absorption in the visible range where the peak is located at $2.70 \mathrm{eV}$ in the zigzag direction and the absorption in the armchair direction is very low [26]. The effect of strain on this anisotropic absorption is demonstrated in Figs. 2(a), 2(b), and 2(c), for uniaxial-armchair, uniaxial-zigzag, and biaxial strain, respectively, where red and green lines specify strains along the armchair and zigzag edges, respectively. It can be seen that the absorption peak shifts mimic the band gap behavior under strain. Therefore, in cases of the armchair and biaxial tensile strain, the absorption peaks shift towards the ultraviolet region of the spectrum with a gradual decrease in the absorption rate, and inversely, compressive strain tends to push the peaks into the infrared zone, while increasing the absorption rates. On the other hand, the strain along the zigzag direction induces an exactly opposite trend, where the tensile strain intensifies the absorption and shifts the peak towards lower energies. As the absorption peak moves towards the infrared region, alongside the initial peak in the visible range, a secondary peak in the infrared region appears.

A comparison between o- $\mathrm{B}_{2} \mathrm{~N}_{2}$ and other $2 \mathrm{D}$ materials with strong optical response highlights the special behavior of o- $\mathrm{B}_{2} \mathrm{~N}_{2}$ upon strain engineering. Black phosphorus, which has a significant absorption in the UV region, shows a considerable shift towards the visible range coupled with flattening of the absorption peaks under strained conditions [49]. It was also found that $\mathrm{H}-\mathrm{MoS}_{2}$, as one of the best candidates for absorption in the visible region, is highly sensitive to strain, showing a redshift in response to strain, a common feature among TMDs $[63,64]$. In contrast, o- $\mathrm{B}_{2} \mathrm{~N}_{2}$ offers a flexible band gap tunability, while the peak of light absorption lies in the visible range in a wide range of uniaxial and biaxial strains. In addition, it preserves its anisotropic absorption behavior independent of the type and scope of the applied strain. Moreover, in comparison to o- $\mathrm{B}_{2} \mathrm{~N}_{2}$, the optical absorption in $\mathrm{o}-\mathrm{B}_{2} \mathrm{P}_{2}, \mathrm{o}-\mathrm{Al}_{2} \mathrm{~N}_{2}$, and o- $\mathrm{Ga}_{2} \mathrm{~N}_{2}$ is weaker, and less anisotropic in the visible range [61].

An efficient photocatalyst for water splitting needs to possess several features simultaneously. First, to have optimum solar light absorption, the band gap energy must be less than $3 \mathrm{eV}$. Second, the band gap width must exceed the free energy of water splitting, equivalent to $1.23 \mathrm{eV}$, to provide sufficient energy to break covalent bonds in water. Third, it needs to have a suitable band edge alignment with the reduction and oxidation potentials of water splitting. In this respect, the VBM location in the energy frame must to be lower than the oxidation potential of water $\left(\mathrm{O}_{2} / \mathrm{H}_{2} \mathrm{O},-5.67 \mathrm{eV}+\mathrm{pH}\right.$ $\times 0.059 \mathrm{eV}$ ), and the CBM position must be higher than the reduction potential of water $\left(\mathrm{H}^{+} / \mathrm{H}_{2},-4.44 \mathrm{eV}+\mathrm{pH}\right.$ $\times 0.059 \mathrm{eV})$. The photoinduced electron-hole pair drift for an average lifetime of $\tau$ before annihilation. To have an efficient photocatalyst, the fourth essential requirement is a prolonged $\tau$. To achieve this final requirement, the photogenerated electron-hole pairs must get separated to prevent their rapid recombination. Semiconductors with anisotropic electron and hole mobilities possess a prolonged charge carrier lifetime.

It can be seen that, in the pristine condition, the $1.7 \mathrm{eV}$ band gap energy of o- $\mathrm{B}_{2} \mathrm{~N}_{2}$ lies within the energy range suitable for the splitting of water. Moreover, due to its intrinsic structural anisotropy, this 2D material offers anisotropic charge carrier mobilities, which increase the charge carrier lifetime. It also has a very promising optical absorption in the visible range of the light spectrum, which covers $43 \%$ of the incident beams, reaching the Earth's surface. The only drawback of o- $\mathrm{B}_{2} \mathrm{~N}_{2}$ is its partial band edge alignment with the redox potential of water splitting. This final deficiency of $o-B_{2} \mathrm{~N}_{2}$ can also be cured through strain engineering. As is shown in Fig. 3, the required band edge alignment occurs under the tensile armchair and biaxial strain. In these two regimes, as the percentage of strain increases, a better band alignment can be achieved. Ultimately, with around 12 and $8 \%$ tensile armchair and biaxial strains, respectively, a perfect band alignment can be obtained. It should be mentioned that tensile zigzag strain and all forms of compressive strains deteriorate the compatibility of band edges with the redox potential of water splitting.

\section{CONCLUSION}

We performed first-principles calculations to investigate the effect of strain on the electronic, structural, and optical properties of $\mathrm{o}-\mathrm{B}_{2} \mathrm{~N}_{2}$. Boron and nitrogen are abundant and cheaply accessible on Earth, and their compounds are highly noble and thermally stable. The well-known h-BN has already dominated the $2 \mathrm{D}$ realm, as the best $2 \mathrm{D}$ insulator option. With a band gap of $1.7 \mathrm{eV}, \mathrm{o}-\mathrm{B}_{2} \mathrm{~N}_{2}$ can offer both the desired features of $\mathrm{h}-\mathrm{BN}$ and a semiconductor crystal. Here, we demonstrated that $\mathrm{o}-\mathrm{B}_{2} \mathrm{~N}_{2}$ possesses high ultimate strain, comparable to that of graphene, which makes strain engineering of this material feasible even in extreme straining regimes. We found that besides exhibiting excellent electronic and optical properties in its pristine condition, o- $\mathrm{B}_{2} \mathrm{~N}_{2}$ can also be an ideal option to achieve any desired band gap up to 

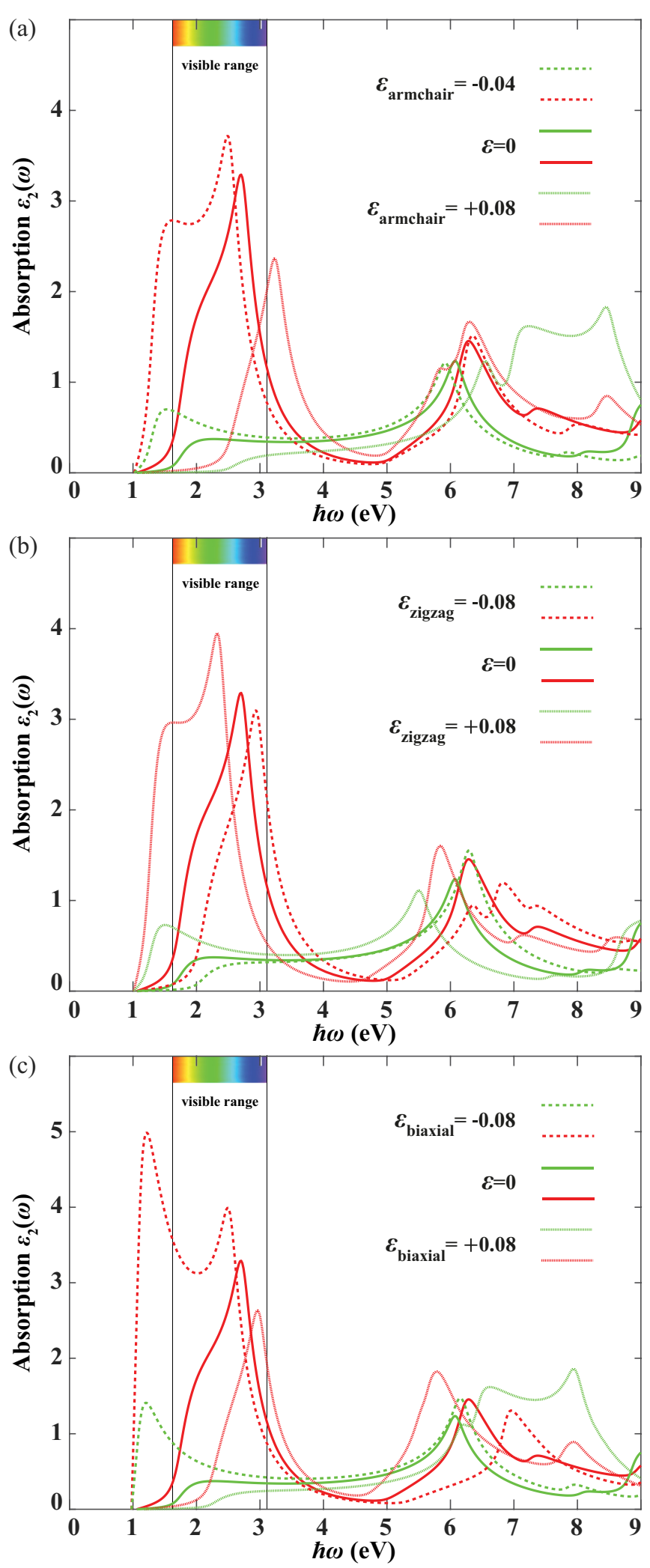

FIG. 2. Direction-dependent optical absorption spectra of o$\mathrm{B}_{2} \mathrm{~N}_{2}$ upon (a) armchair, (b) zigzag, and (c) biaxial strain engineering. Red and green lines show the light absorption along armchair and zigzag directions, respectively. In (a), the -0.04 case is provided instead of the -0.08 case, since the latter offers much less absorption in the visible range.
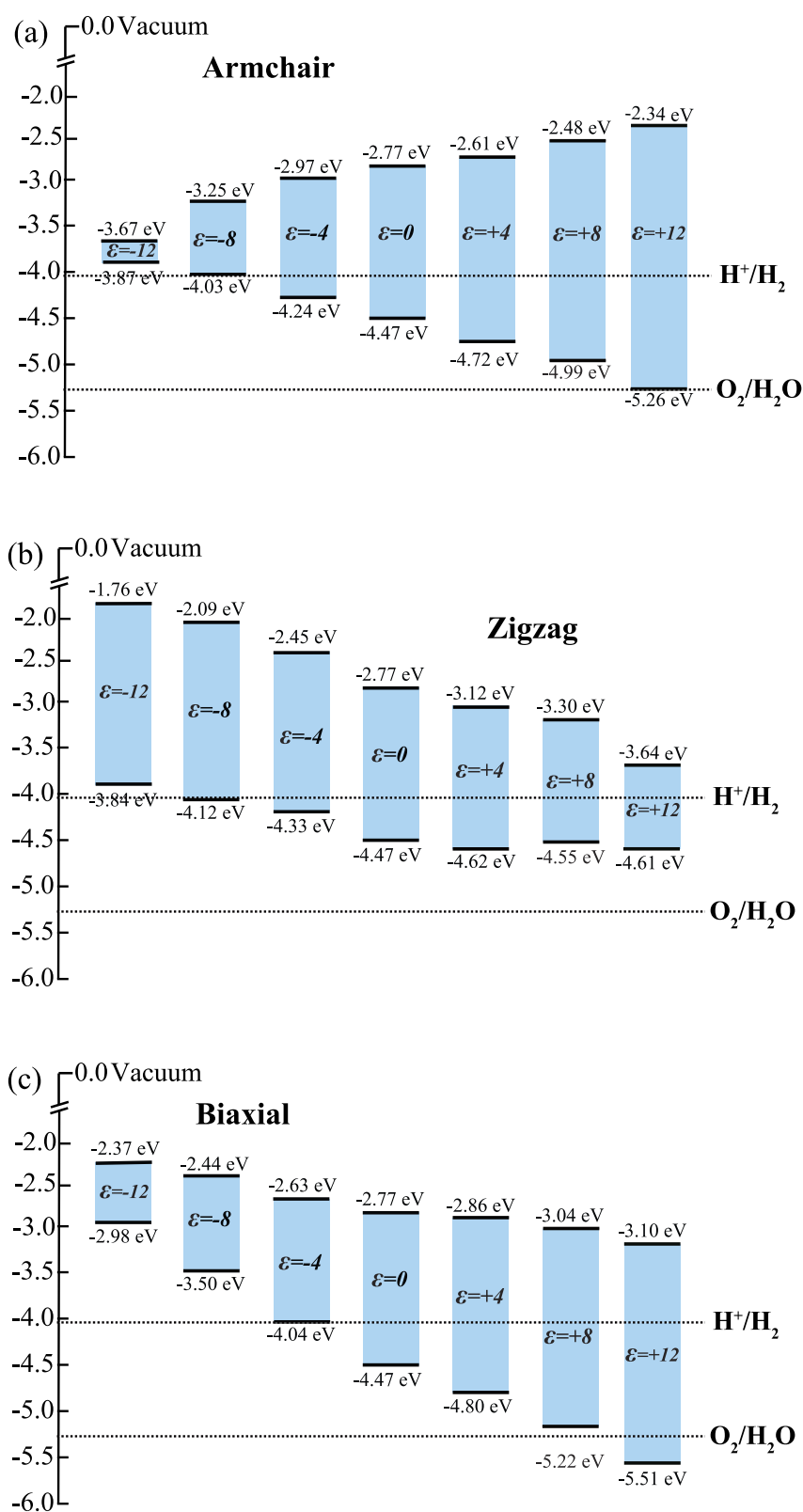

FIG. 3. Location of the band edges (CBM and VBM) in o- $\mathrm{B}_{2} \mathrm{~N}_{2}$ with respect to the vacuum level obtained from the HSE06 functional, where (a), (b), and (c) are related to armchair, zigzag, and biaxial strain, respectively. Strain values are denoted in percent. The dash-dotted lines are aligned with the oxidation and reduction potentials of water splitting, where $\mathrm{pH}=7$.

$2.92 \mathrm{eV}$ while preserving the optimal optical absorption rate, through strain engineering. In fact, the scope of strain tunability and anisotropic tunability found in $\mathrm{o}-\mathrm{B}_{2} \mathrm{~N}_{2}$ is rare among $2 \mathrm{D}$ materials. Although, in the pristine condition, $\mathrm{o}-\mathrm{B}_{2} \mathrm{~N}_{2}$ has a wide enough band gap for application in photocatalytic water splitting, its band edges are only partially aligned with the redox potential of water splitting. Via strain engineering, however, the position of band edges can be tailored to become perfectly aligned with the redox potential. Having a suitable band gap, excellent optical absorption in the visible range, and anisotropic carrier mobilities which prolong the average 
excitonic lifetime, upon strain engineering of the band edges, $\mathrm{o}-\mathrm{B}_{2} \mathrm{~N}_{2}$ can turn into a promising candidate for applications as a photocatalyst in the water-splitting process.

\section{ACKNOWLEDGMENTS}

This work was supported by the Scientific and Technological Research Council of Turkey (TÜBİTAK) under Project
No. 118F097. S.J. acknowledges support from the Turkish Academy of Sciences-Outstanding Young Scientists Award Program (TÜBA-GEBIP). Part of the computational resources is provided by the National Center for High Performance Computing of Turkey (UHeM) under Grant No. 5003622015. S.D. thanks UNAM, National Nanotechnology Research Center at Bilkent University, for the hospitality.

S.D. and S.E.R. contributed equally to this work.
[1] K. S. Novoselov, A. K. Geim, S. V. Morozov, D. Jiang, Y. Zhang, S. V. Dubonos, I. V. Grigorieva, and A. A. Firsov, Science 306, 1102896 (2004).

[2] K. S. Novoselov, A. K. Geim, S. V. Morozov, D. Jiang, M. I. Katsnelson, I. V. Grigorieva, S. V. Dubonos, and A. A. Firsov, Nature (London) 438, 197 (2005).

[3] Y. Zhang, Y. Tan, H. L. Stromer, and P. Kim, Nature (London) 438, 201 (2005).

[4] A. K. Geim and K. S. Novoselov, Nat. Mater. 6, 183 (2007).

[5] F. Schwierz, Nat. Nanotechnol 5, 487 (2010).

[6] K. F. Mak, M. Y. Sfeir, Y. Wu, C. H. Lui, J. A. Misewich, and T. F. Heinz, Phys. Rev. Lett. 101, 196405 (2008).

[7] H. Liu, Y. Liu, and D. Zhu, J. Mater. Chem. 21, 3335 (2011).

[8] M. Topsakal and S. Ciraci, Appl. Phys. Lett. 98, 131908 (2011).

[9] J. Wang, F. Ma, W. Liang, and M. Sun, Mater. Today Phys. 2, 6 (2017).

[10] J. O. Sofo, A. S. Chaudhari, and G. D. Barber, Phys. Rev. B 75, 153401 (2007).

[11] T. M. G. Mohiuddin, A. Lombardo, R. R. Nair, A. Bonetti, G. Savini, R. Jalil, N. Bonini, D. M. Basko, C. Galiotis, N. Marzari, K. S. Novoselov, A. K. Geim, and A. C. Ferrari, Phys. Rev. B 79, 205433 (2009).

[12] Y. Wang, C. Cong, W. Yang, J. Shang, N. Peimyoo, Y. Chen, J. Kang, J. Wang, W. Huang, and T. Yu, Nano Res. 8, 2562 (2015).

[13] G. Plechinger, F. X. Schrettenbrunner, J. Eroms, D. Weiss, C. Schüller, and T. Korn, Phys. Status Solidi RRL 6, 126 (2012).

[14] Y. Y. Hui, X. Liu, W. Jie, N. Y. Chan, J. Hao, Y. Hsu, L. Li, W. Guo, and S. P. Lau, ACS Nano 7, 7126 (2013).

[15] F. Li, T. Shen, C. Wang, Y. Zhang, J. Qi, and H. Zhang, Nano-Micro Lett. 12, 106 (2020).

[16] T. Yu, Z. Ni, C. Du, Y. You, Y. Wang, and Z. Shen, J. Phys. Chem. C 112, 12602 (2008).

[17] W. H. Chae, J. D. Cain, E. D. Hanson, A. A. Murthy, and V. P. Dravid, Appl. Phys. Lett. 111, 143106 (2017).

[18] S. Yang, Y. Chen, and C. Jiang, InfoMat 3, 397 (2021).

[19] H. H. Perez Garza, E. W. Kievit, G. F. Schneider, and U. Staufer, Nano Lett. 14, 4107 (2014).

[20] G. Gui, J. Li, and J. Zhong, Phys. Rev. B 78, 075435 (2008).

[21] M. Topsakal, S. Cahangirov, and S. Ciraci, Appl. Phys. Lett. 96, 091912 (2010).

[22] S. Horzum, H. Sahin, S. Cahangirov, P. Cudazzo, A. Rubio, T. Serin, and F. M. Peeters, Phys. Rev. B 87, 125415 (2013).

[23] L. Liu, Y. P. Feng, and Z. X. Shen, Phys. Rev. B 68, 104102 (2003).

[24] K. Watanabe, T. Taniguchi, and H. Kanda, Nat. Mater 3, 404 (2004).

[25] L. Wirtz, A. Marini, and A. Rubio, in Electronic Properties of Novel Nanostructures: XIX International
Winterschool/Euroconference on Electronic Properties of Novel Materials, AIP Conference Proceedings Vol. 786 (American Institute of Physics, Melville, NY, 2005), p. 391.

[26] S. Demirci, S. E. Rad, S. Kazak, S. Nezir, and S. Jahangirov, Phys. Rev. B 101, 125408 (2020).

[27] Z. Guo, J. Zhou, and L. Zhu, J. Mater. Chem. A 4, 11446 (2016).

[28] A. K. Singh, K. Mathew, H. L. Zhuang, and R. G. Hennig, J. Phys. Chem. Lett. 6, 1087 (2015).

[29] B. Luo, G. Liu, and L. Wang, Nanoscale 8, 6904 (2016).

[30] Y. Sun, Z. Sun, S. Gao, H. Cheng, Q. Liu, J. Piao, T. Yao, C. Wu, S. Hu, S. Wei, and Y. Xie, Nat. Commun. 3, 1057 (2012).

[31] Y. Sun, H. Cheng, S. Gao, Z. Sun, Q. Liu, Q. Liu, F. Lei, T. Yao, J. He, S. Wei, and Y. Xie, Angew. Chem. 51, 8727 (2012).

[32] Y. Xu, W. Zhao, R. Xu, Y. Shia, and B. Zhang, Chem. Commun. 49, 9803 (2013).

[33] D. Voiry, H. Yamaguchi, J. Li, R. Silva, D. C. B. Alves, T. Fujita, M. Chen, T. Asefa, V. B. Shenoy, G. Eda, and M. Chhowalla, Nat. Mater. 12, 850 (2012).

[34] P. E. Blöchl, Phys. Rev. B 50, 17953 (1994).

[35] J. P. Perdew, K. Burke, and M. Ernzerhof, Phys. Rev. Lett. 77, 3865 (1996).

[36] H. J. Monkhorst and J. D. Pack, Phys. Rev. B 13, 5188 (1976).

[37] G. Kresse and J. Furthmüller, Phys. Rev. B 54, 11169 (1996).

[38] A. V. Krukau, O. A. Vydrov, A. F. Izmaylov, and G. E. Scuseria, J. Chem. Phys. 125, 224106 (2006).

[39] H. Ehrenreich and M. H. Cohen, Phys. Rev. 115, 786 (1959).

[40] H. Sun, S. Mukherjee, and C. V. Singh, Phys. Chem. Chem. Phys. 18, 26736 (2016).

[41] Q. Peng, W. Ji, and S. De, Comput. Mater. Sci. 56, 11 (2012).

[42] C. C. Liu, W. Feng, and Y. Yao, Phys. Rev. Lett. 107, 076802 (2011).

[43] Y. Cai, C. P. Chuu, C. M. Wei, and M. Y. Chou, Phys. Rev. B 88, 245408 (2013).

[44] T. Kaloni and U. Schwingenschlogl, Chem. Phys. Lett. 583, 137 (2013).

[45] D. R. Kripalani, A. A. Kistanov, Y. Cai, M. Xue, and K. Zhou, Phys. Rev. B 98, 085410 (2018).

[46] L. Wang, A. Kutana, and B. I. Yakobson, Ann. Phys. (Berlin) 526, 9 (2014).

[47] M. Ghorbani-Asl, S. Borini, A. Kuc, and T. Heine, Phys. Rev. B 87, 235434 (2013).

[48] E. Scalise, M. Houssa, G. Pourtois, V. Afanasev, and A. Stesmans, Nano Res. 5, 43 (2011).

[49] D. Cakir, H. Sahin, and F. M. Peeters, Phys. Rev. B 90, 205421 (2014).

[50] H. Liu, A. T. Neal, Z. Zhu, Z. Luo, X. Xu, D. Tomnek, and P. D. Ye, ACS Nano 8, 4033 (2014). 
[51] X. Peng, Q. Wei, and A. Copple, Phys. Rev. B 90, 085402 (2014).

[52] J. Wu, B. Wang, Y. Wei, R. Yang, and M. Dresselhaus, Mater. Res. Lett. 1, 200 (2013).

[53] Z. G. Shao, X. S. Ye, L. Yang, and C. L. Wang, J. Appl. Phys. (Melville, NY) 114, 093712 (2013).

[54] J. Wiktor and A. Pasquarello, Phys. Rev. B 94, 245411 (2016).

[55] Y. Wang and Y. Ding, Nanoscale Res. Lett. 10, 254 (2015).

[56] Q. Wei, G. Yang, and X. Peng, Phys. Rev. Appl. 13, 034065 (2020).

[57] M. E. Kilic and K. Lee, Carbon 174, 368 (2021).
[58] M. E. Kilic and K. Lee, Nanoscale 13, 9303 (2021).

[59] H. Lang, S. Zhang, and Z. Liu, Phys. Rev. B 94, 235306 (2016).

[60] A. Rawat, N. Jena, Dimple, and A. De Sarkar, J. Mater. Chem. A 6, 8693 (2018).

[61] J. Zhao, H. Zeng, and G. Yao, Phys. Chem. Chem. Phys. 23, 3771 (2021).

[62] S. M. Sze and K. K. Ng, Physics of Semiconductor Devices (Wiley, New York, 1981).

[63] H. J. Conley, B. Wang, J. I. Ziegler, R. F. Haglund, S. T. Pantelides, and K. I. Bolotin, Nano Lett. 13, 3626 (2013).

[64] K. He, C. Poole, K. F. Mak, and J. Shan, Nano Lett. 13, 2931 (2013). 